\title{
Planar Scanning Plane Comprehensive Error Data Measurement Analysis
}

\author{
Liu jun ${ }^{1, a}$, Tu chao ${ }^{2, b}$ \\ ${ }^{1}$ Basic Department, Changchun Institute of Engineering and Technology, china \\ ${ }^{2}$ Vehicle Engineering Department, Changchun Institute of Engineering and Technology, china \\ a332552083@qq.com, bfdn_198591@163.com,
}

Keywords: scanning plane,Laser Tracker,error identification

\begin{abstract}
In this paper,the positioning data for large planar scanning plane were measured with laser tracker, converted the coordinate by spatial error between scanning plane and tracker , and analyzed the converted error data. For the subsequent use of the least squares method error compensation provided.
\end{abstract}

\section{Identification of Measurement Error}

Comprehensive error measurement data identification method is through a variety of measurements, scanned plane scanning plane position measurement position error parameter. After identification of the mathematical model of its integrated error parameters measured, indirectly discrete values scanning plane of the error parameter.

Laser Tracker is the most common stage of a measurement of space target tracking and real-time measurement of three-dimensional coordinates of the target space. By coordinate transformation can be determined from the deviation and the angle deviation anchor points. Simultaneous measurement of high precision, easy operation, broken light Renewed good.

Measuring principle is shown below:

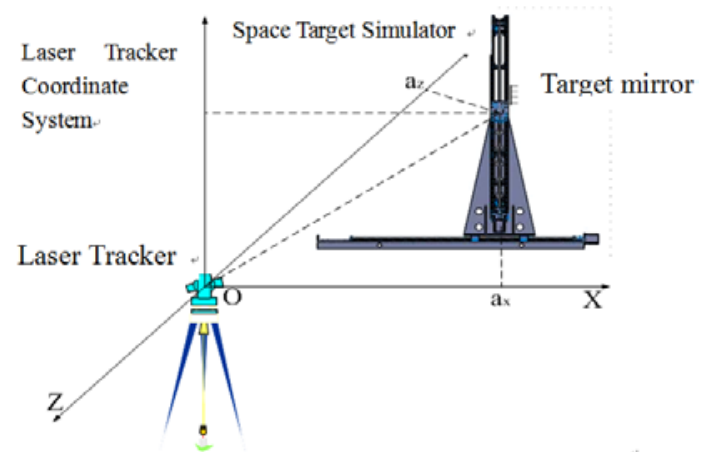

Fig1 Test system schematic

The laser beam passes through the mirror is mounted on a target object to be measured (scanning plane probe position) reflected laser tracker emitted by laser interference principle can achieve three-dimensional coordinate measuring three-dimensional space coordinates of any point. According to this feature, the rotational center of the laser tracker as the origin of the space coordinates, respectively, in the direction of scanning plane XYZ-axis rail is defined as the XYZ coordinate system, the center of the sphere as a target by a mirror measuring point. The coordinates of the target mirror in space as three-dimensional coordinates is $\left(a_{x}, a_{y}, a_{z}\right)$. As the target is moved in the scanning $\mathrm{X}, \mathrm{Y}$ plane, laser tracker available three-dimensional coordinates of each mobile points. We could measure and evaluate the surface flatness data which was from scanning $\mathrm{X}, \mathrm{Y}$ plane.

The establishment of reference coordinate system and scanning plane coordinate system is not strictly coincide, therefore coordinate error exists between scanning plane coordinate system $O^{\prime}-X^{\prime} Y^{\prime} Z^{\prime}$ and the reference coordinate system $O-X Y Z$. 


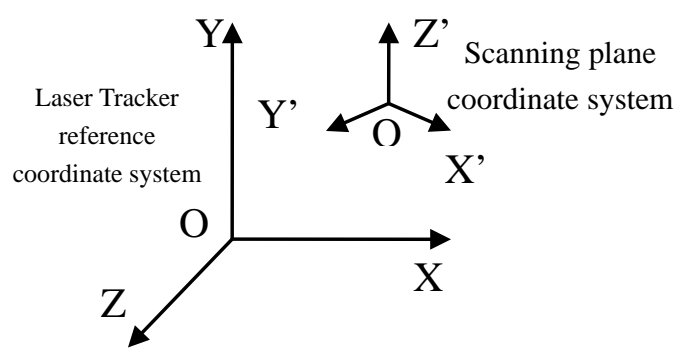

Fig 2

According to the error model, two coordinates corresponding coordinate the following correspondence:

$$
\left[\begin{array}{l}
x \\
y \\
z
\end{array}\right]=\Delta T \cdot\left[\begin{array}{l}
x^{\prime} \\
y^{\prime} \\
z^{\prime}
\end{array}\right]
$$

Where $(\mathrm{x}, \mathrm{y}, \mathrm{z})$ is the reference coordinate system coordinates, (x ', $\mathrm{y}^{\prime}, \mathrm{z}$ ') is scanning plane coordinate system coordinates.

When we know the two points' $(A, B)$ coordinates in the $(O-X Y Z)$ and $\left(O^{\prime}-X^{\prime} Y^{\prime} Z^{\prime}\right)$ are (xA, $\mathrm{yA}, \mathrm{zA}),\left(\mathrm{xA}^{\prime}, \mathrm{yA}^{\prime}, \mathrm{zA}^{\prime}\right)$ and $(\mathrm{xB}, \mathrm{yB}, \mathrm{zB}),\left(\mathrm{xB}^{\prime}, \mathrm{yB}^{\prime}, \mathrm{zB}^{\prime}\right)$ then the three attitude angle $[\alpha, \beta, \gamma]$ and three displacement amount $[\Delta x, \Delta y, \Delta z]$ can be obtained.

$$
\delta_{0}=\left[\begin{array}{l}
\Delta X \\
\Delta Y \\
\Delta Z
\end{array}\right]=\llbracket\left[\begin{array}{l}
x \\
y \\
z
\end{array}\right]-\left[\begin{array}{l}
x^{\prime} \\
y^{\prime} \\
z^{\prime}
\end{array}\right]
$$

For example, experiments, select coordinates of the two test points are: A '[0, 0, 0], A [-0.1543, $-0.0131,-0.0083]$, and B' [2900, 1900, 0], B [2900.257, 1900.124, 0.3461], Numerical units are mm. The results are in (3) and (4) as follows:

$$
\begin{aligned}
& {\left[\begin{array}{l}
\alpha \\
\beta \\
\gamma
\end{array}\right]=\left[\begin{array}{c}
3.666^{\circ} \\
-2.392^{\circ} \\
-113.612^{\circ}
\end{array}\right]} \\
& {\left[\begin{array}{l}
\Delta x \\
\Delta y \\
\Delta z
\end{array}\right]=\left[\begin{array}{c}
-0.1543 \\
-0.0131 \\
-0.0083
\end{array}\right]}
\end{aligned}
$$

\section{Data collection and analysis}

Scanning options: The starting point of the target mirror is the target simulator coordinate zero. Travel locus shown in Figure 3. X-axis and Y-axis are 100mm intervals collection. X-axis travel of total $2900 \mathrm{~mm}$, Y-axis travel of $1900 \mathrm{~mm}$ total. Measuring a total of 600 points, the scanning speed of scanning plane $30 \mathrm{~mm} / \mathrm{s}$. 


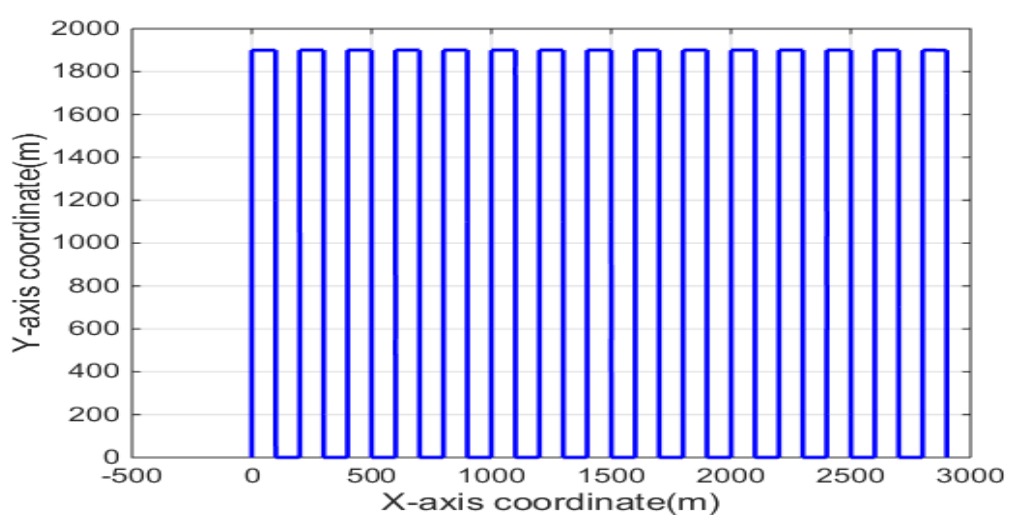

Fig 3 Field scanning program

Laser tracker get the coordinates data after several experiments scanning. Table 1 show some of the data in which the first test (unit: $\mathrm{mm}$ )

Table 1 Some of the test data

\begin{tabular}{ccccccccc}
\hline $\mathrm{X}$ & 0 & -0.0622 & -0.0403 & -0.0618 & -0.1423 & -0.1719 & -0.1408 & -0.0991 \\
\hline $\mathrm{Y}$ & 0 & 99.9829 & 199.9952 & 299.9983 & 399.9858 & 499.9822 & 600.0263 & 700.0549 \\
$\mathrm{Z}$ & 0 & -0.013 & 0.0084 & 0.0098 & -0.0031 & 0.0091 & 0.0144 & 0.0186 \\
\hline
\end{tabular}

Through the data, we get a three-dimensional coordinate grid map of the target mirror. Shown in Figure 4

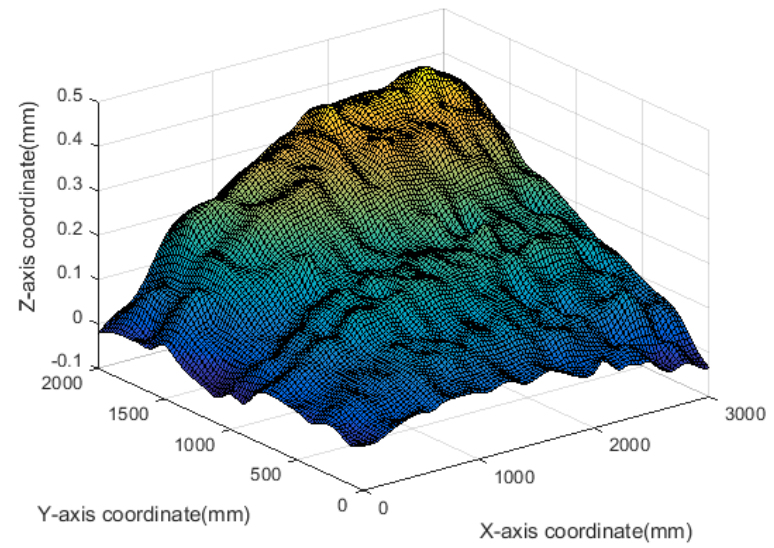

Fig 4 three-dimensional coordinate grid map

Figure 4 Analysis: the Z-axis coordinate of the test point away from of origin is large, the measurement accuracy is low, flatness is poor; the Z-axis coordinate of the test point near the origin is small, good flatness, high measurement accuracy.

Distributions of the Z-axis coordinate values shown in Figure5.

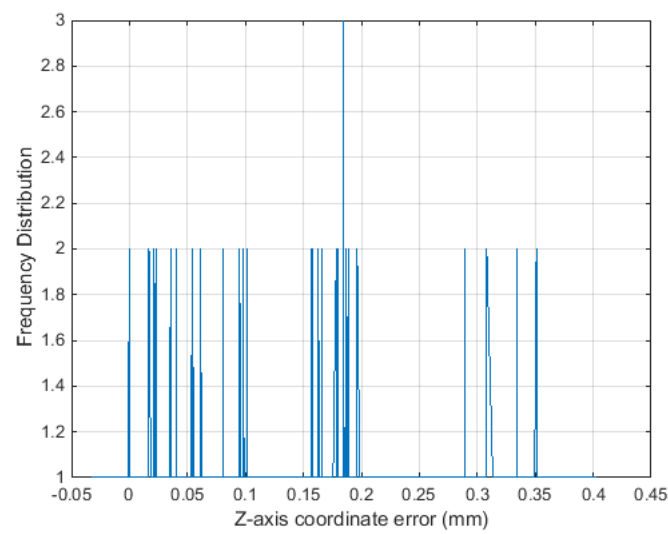

Fig 5 Z-axis coordinate distribution 
Figure 5 Analysis: Z-axis coordinate value distribution [-0.05mm $\sim 0.35 \mathrm{~mm}]$ range, in the [0mm $\sim 0.2 \mathrm{~mm}$ ] interval distribution is most concentrated. The histogram is similar with the distribution of this segment of the rectangle. The standard deviation of formula as follows (5):

$$
s=\sqrt{\frac{\sum_{i=1}^{n}\left(z_{i}-\bar{z}\right)}{n-1}}=0.012 \mathrm{~mm}
$$

In the formula (5), $\mathrm{n}$ =number of measuring points; $\mathrm{zi}=\mathrm{Z}$ coordinates of each measuring point; $\mathrm{z}$ $=$ the average Z-axis coordinates;

Based on the above analysis, due to different system errors and other unknown factors combined effects, Laser Tracker obtained different residuals of Z-axis coordinate data at different locations. The data showing the characteristics of the non-Precision Measurement.We use each of the square of reciprocal of the residuals data of Z-axis coordinate as the weights of each measurement point data. It ensures high accuracy, good flatness coordinate data precision flatness contribution to high demand. For coordinate offset by the weighted least squares method provides a good basis.

\section{Reference}

[1] Li kai, Hu yinghui,Yuan feng.Simulator flatness measurement and compensation of Spatial Target Based on weighted least squares [J].Optical Electronic Engineering,2015（04）.

[2] Zhang fushun.Error Analysis and Compensation ultralow sidelobe antenna planar near field measurement [J].Xi'an Electronic Science and Technology (doctoral thesis),1999 (12).

[3] Wng xiaokun. Using a laser tracker measuring straightness long way [J]. Applied Optics, 2013, 34(4).

[4] Liu chengyu, Li haolin. Straightness space is based on the principle of coordinate conversion error evaluation [J]. Modern Manufacturing Engineering, 2013(3). 